\title{
Preparing Teachers to Infuse Computational Science into their Classroom Instruction
}

\author{
Susan J. Ragan \\ Maryland Virtual High \\ School \\ 14217 Bradshaw Drive \\ Silver Spring, MD 20905 \\ 301-989-0151 \\ mvhsragan@gmail.com
}

\author{
Cheryl Begandy \\ Pittsburgh \\ Supercomputing Center \\ 300 S. Craig Street \\ Pittsburgh, PA 15213 \\ 412-268-5129 \\ begandy@psc.edu
}

\author{
Nancy R. Bunt \\ Math \& Science \\ Collaborative \\ Allegheny Intermediate \\ Unit \\ 475 E. Waterfront Dr. \\ Homestead, PA 15120 \\ 412-394-4598 \\ nancy.bunt@aiu3.net
}

\author{
Charlotte M. Trout \\ Washington County Public \\ Schools \\ 820 Commonwealth Ave. \\ Hagerstown, MD 21740 \\ 301-766-8790 \\ troutcha@wcboe.k12.md.us
}

\author{
Scott A. Sinex \\ Prince George's \\ Community College \\ 301 Largo Road \\ Largo, MD 20774 \\ 301-341-3023 \\ ssinex@pgcc.edu
}

\begin{abstract}
Establishing consistent use of computer models and simulations in $\mathrm{K}-12$ classrooms has been a challenge for the computational science education community. Scaling successful local efforts has been particularly difficult. In this article we describe how a training model from one place and time can be translated into a training model for another very different place and time if critical factors such as school system culture, professional development organization, local learning standards and goals, and collaboration between STEM disciplines are taken into account.
\end{abstract}

\section{Categories and Subject Descriptors \\ K.3 Computers in Education}

\section{General Terms}

Management, Design, Human Factors

\section{Keywords}

Secondary Science Education, Computer Models, Simulations, Professional Development Program

Permission to make digital or hard copies of all or part of this work for personal or classroom use is granted without fee provided that copies are not made or distributed for profit or commercial advantage and that copies bear this notice and the full citation on the first page. To copy otherwise, or republish, to post on servers or to redistribute to lists, requires prior specific permission and/or a fee. Copyright (CJOCSE, a supported publication of the Shodor Education Foundation Inc.

\section{INTRODUCTION}

A recent publication by the National Academy Press, Learning Science through Computer Games and Simulations, [1] devotes a chapter to the question of how to promote the use of computer simulations in science classrooms. While acknowledging that research on their effectiveness still needs additional input, they note some of the obstacles to the consistent adoption of computer simulations and games in K12 classrooms. One of the constraints cited has to do with the fact that teachers do not always possess the content knowledge and teaching strategies needed to achieve the full potential of the simulation.

The Maryland Virtual High School of Science and Mathematics (MVHS) has over seventeen years of experience working with Maryland teachers to help them use computer models and simulations as tools to assist their students' comprehension of complex concepts. In 2006, MVHS was invited by the Pittsburgh Supercomputing Center (PSC) to bring its training model to the Pittsburgh area. Four years later the Math \& Science Collaborative (MSC) joined the effort to bring computational thinking and reasoning into science and math classrooms in southwestern Pennsylvania. Through our experiences in two neighboring states, we have found that school system culture must be taken into account when developing workshops for teacher training in computational science. In this article, we describe the approaches we have used and the lessons we have learned. The setting for our work in Maryland was 24 school districts which are county or city-wide and where statewide learning goals in science began to influence local instruction in the mid-90's. In contrast, in Pennsylvania, 500 local school districts are served by 29 intermediate units, which are regional educational service agencies covering the 67 counties. Statewide standards in science were also established in the $90 \mathrm{~s}$ in Pennsylvania. Both states are signatories to the new Common Core State Standards [2] in mathematics and language arts - which will increase the potential for transfer of learning from one state to another.

\section{BACKGROUND}

\subsection{Maryland Virtual High School}

MVHS [3] has been working with teachers since 1994 when its first grant from the National Science Foundation (NSF) was awarded. Over its lifetime, MVHS has demonstrated methods by which science teachers can integrate computational science projects into their classrooms and, in the process, provide students with a modern and compelling introduction to the concepts of 
science through inquiry-based activities while supporting both state and national standards for content. In the beginning, it was challenging to get schools involved in computational science since Internet connectivity was slow or nonexistent and there were barely any secondary school-level appropriate applications available. On the other hand, standardized testing had not yet taken a stranglehold on the curriculum so innovative teachers were free to try new things and their principals were supportive of their efforts.

During MVHS's first ten years, the focus was on preparing teachers to not only use and build computer models and simulations to help teach science concepts, but also to become trainers themselves so they could disseminate these new methods to their colleagues. A community of teachers who were comfortable assisting one another as coaches in the classroom was developed [4]. According to the final evaluation of MVHS's second NSF grant, CoreModels [5], the successes of MVHS were due to these key factors [6]:

- The close-knit, peer-to-peer structure of the CoreModels community of teachers

- The presence of a core group of relatively senior and especially dedicated teachers who were able to act as a vanguard in exploring the relative value of studentdriven model construction and open-ended inquiry into systems

- Its sustained, long-term commitment to curriculum development, testing and revision

After CoreModels ended, MVHS used its funding through various partners to support the CoreModels teachers as they conducted workshops in their own school districts. Although the workshop evaluations from the teachers were overwhelmingly positive, resources were inadequate to determine how much computational science was actually being infused into the classroom outside of our CoreModels group. Anecdotal information implied that the impact of the computational science innovation varied significantly from one district to another.

\subsection{Computation and Science for Teachers}

In 2006, members of the MVHS team were invited to work with the Pittsburgh Supercomputing Center to develop a "Core Models" type of program within the Pittsburgh area schools. Using MVHS's "train the trainer" model, a series of one-week summer workshops with follow-up sessions during the school year were developed and delivered. Known as Computation and Science for Teachers (CAST) [7], three cohorts of teachers benefitted from the program and responded enthusiastically to the CAST workshops. The following comments are representative:

- “...this workshop provided me with more ideas on how to infuse technology in my lessons..."

- “...I saw connections between data collection and modeling, and better connection between math and science..."

- “...I am now thinking more about mathematical modeling, not just physical model...I plan to model more astronomy and chemistry concepts..."

Here are the words of one teacher on how CAST affected his teaching and his students:

"The CAST experience has been an invaluable asset in my teaching evolution. Initially it began as a completely utilitarian device that allowed me to provide students with an alternative to missed lab work, and then I began to explore the possibilities of doing labs that were either too costly or too dangerous to perform in a high school environment, but it has grown into something more...

Systems modeling has fundamentally altered the way I teach, and not with just software. At the heart of systems modeling is the notion of connections. The examination of the connections can be done independent of the application software without losing the scientific and educational relevance. Demonstrating to a young mind the relationships that exist in nature without the cloud of mathematical complexity is crucial in the development of a scientifically literate generation."

While the enthusiasm of these teachers is encouraging and many teachers did develop lessons to use with their students, the overall impact of this approach on student learning was difficult to measure and continued use by teachers has been limited. The fact that few teachers elected to return to the project for advanced training in the second and third years made the MVHS leaders question their approach. Is it possible for a traveling team of trainers (MVHS) to provide enough support to get a true learning community off the ground in another state? How can MVHS facilitate the integration of the knowledge and skills of the various players? The PSC has a vested interest in helping the local math and science education community embrace computational science, but has limited funding to do so. MVHS leaders are willing to share their expertise with others outside Maryland; but, clearly, that is not enough. In this article, the lessons learned from the adaptation of the Maryland approach to one that better fit southwestern Pennsylvania will be examined.

\section{LESSONS LEARNED}

\subsection{Understand the State's Public Education Structure}

Maryland is based on a county/city-wide system of public schools which means that most school districts have multiple secondary schools whose teachers are accustomed to meeting and collaborating with their peers. Pennsylvania, on the other hand, has over 500 school districts that have a long history of operating independently. Some districts are so small that a single teacher has responsibility for all biology or all chemistry or even all science classes at the high school level. These structural differences lead to school culture differences which affect the implementation of innovation as the examples below will illustrate.

In 1994, when the MVHS project was started, the Maryland State Department of Education (MSDE) was already discussing the establishment of core learning goals for science and other subjects [8]. When MVHS leaders went to MSDE with a proposal to bring Internet connectivity to several high schools for the purpose of improving science instruction, MSDE gave its whole-hearted support. Since Maryland is based on a county/city-wide model for school districts, it was relatively easy to contact 24 superintendents to explain the project to them. Sixteen middle and high schools in thirteen school districts [9] agreed to participate in a three year project in which Internet connectivity would be established at the school and two or three teachers from the school 
would receive in-depth training in the use of the Internet as a collaborative tool for science.

At the end of the three years, the participating teachers were convinced that Internet connectivity was important, but they weren't sure how to use computational science in their classrooms. They had used their connectivity to collaborate with each other on projects such as the virtual earthquake, the boiling point project, and the Eratosthenes project [10]; but they admitted that sustaining such collaboration beyond the end of the project was unlikely. Those outcomes led to the formation of the MVHS CoreModels Project, another three-year NSF-funded project, whose mission was to prepare teachers to create and use computer models in their classroom teaching.

The CoreModels project included 27 high schools from 14 of the 24 school districts in Maryland. In 1997, there weren't many ready-made models for high school science, so project goals included creating a repository of models and lesson plans that would address the Maryland State Science Core Learning Goals [8]. To enable a system of local support for the teachers, the state of Maryland was divided into 3 regions, each with a lead teacher who was released half-time from classroom responsibilities to provide on-site assistance to the other teachers. In the first year of the project, summer workshops and quarterly Saturday workshops were held in a central location so that everyone could get to know one another and establish a basic level of competency. During the second and third years, the lead teachers conducted after-school and summer workshops in their own regions to encourage the development of learning communities where teachers could collaborate and learn from one another.

The collaboration between MVHS and the PSC began in 2006 with meetings to establish goals and analyze the availability of resources and funding. The PSC was very knowledgeable about the politics of their local schools so they carefully selected the school districts to be invited to the CAST project. Each district superintendent or designated representative attended a kick-off meeting where MVHS presented the objectives of the training and urged them to recruit two math/science teachers to attend the training with the goal of becoming a resource for the district. The ten teachers who attended the first year of training [11] were an excellent group of educators, but only two of them returned for further training. The CAST team hypothesized that the lack of local representatives who could travel to the schools to observe the teachers in action was a major reason for a lack of cohesiveness among the group members.

The second and third cohorts of teachers were recruited in various ways, including through the MSC [12], a regional organization charged with providing professional development for math and science instruction throughout western Pennsylvania. Again, these were excellent educators who enthusiastically embraced the computer models we showed them, but the CAST team had difficulty maintaining contact with them. Why was that? What were the differences between Pennsylvania and Maryland?

After much discussion, we concluded that the small, independent school district structure in Pennsylvania leads to a heightened sense of individualism, autonomy, and isolation on the part of the teachers. Having only one or two teachers trained in a number of geographically dispersed districts made the formation of any type of CAST "learning community" almost impossible. Without the support of peers, the teachers were challenged in just feeling comfortable using the new techniques, let alone teaching others. And when schools are so small that there is only one physics teacher (or chemistry or biology) and teachers have not been encouraged to collaborate within their own buildings, it is quite a culture shift to suggest that those same teachers become peer trainers for others. In Maryland, the county-wide school systems meant that subject-area teachers across the high schools in the district were expected to follow the same curriculum. In Pennsylvania, subject area teachers in the high school were likely to be free to teach their subject as they saw fit. Once we admitted that replicating the Maryland CoreModels experience in Pennsylvania was impossible, we were free to think more creatively. We realized that the key to working with teachers in Pennsylvania was to take advantage of the existing professional development infrastructure, the Math \& Science Collaborative, which leads us to the next lesson.

\subsection{Leverage the Professional Development Program at the Local or State Level}

In Maryland, the school districts are large enough to have their own central office-based professional development staff who are responsible for providing training for teachers. Often these teacher trainers are master teachers who have been encouraged to leave classroom teaching to share their expertise with others. Since Pennsylvania has so many small school districts that cannot afford their own dedicated professional development staff, twenty-nine regional training sites have been established to provide professional development to their schools. The examples below illustrate how important it is to include the leaders of the professional development program in any teacher training initiative.

During the MVHS CoreModels project, the participating teachers were encouraged to keep their department heads and science supervisors informed of changes they were making in their classrooms. Some of the teachers enjoyed a great deal of support from their central office, others worked in a laissez-faire environment, and some found their supervisors to be almost hostile. In retrospect, it is clear that the CoreModels leadership needed to pay more attention to those instructional leaders to be sure that they felt valued and informed of the benefits coming into their schools.

However, those teachers who did enjoy the support of their districts were able to make a significant impact in their counties. In St. Mary's County, a CoreModels biology teacher rose to become the science coordinator where she was influential in infusing technology and computer modeling into the science curriculum across her district. A CoreModels physics teacher in the same county led many district-supported workshops for the teachers in his district and was so inspirational to his students that he was selected the 2011 Teacher of the Year for his school district [13]. In Anne Arundel County, a CoreModels physics teacher trained other teachers in his county even after the CoreModels project ended. When he retired from full-time teaching, he became involved in a NASA-funded project to bring systems modeling into a freshman engineering course in the county. In Montgomery County, a CoreModels biology teacher was hired by the Maryland State Department of Education where he has promoted the use of computer models across the science curriculum.

Washington County (WCPS), a semi-rural school district serving over 21,000 students (pre-K - grade 12), has experienced the most widespread use of computer models across secondary science classrooms due to the intersection of two important people - a CoreModels teacher with superior content and leadership skills 
and a science supervisor with vision and patience. Like most school districts, WCPS educators struggled with finding the most effective ways to use the computer technology that fills their buildings. Fortunately, strong and consistent leadership in science instruction at the district level has resulted in a climate in which secondary science teachers are provided high-quality training and support. In particular, since the science supervisor understands the value of simulations as one tool among many to help students master science concepts, she provides both workshops and inclass support for the teachers as they learn to seamlessly integrate computer models in their teaching. While the CoreModels teacher was still full-time in the classroom, she led most of the summer workshops. Now that she is an instructional specialist in the science supervisor's office, she is able to work with teachers directly in their classrooms, even designing custom simulations to meet a teacher's needs [14]. The benefit of this partnership is that while the classroom teacher is building confidence in the effective use of the simulation, $\mathrm{s} / \mathrm{he}$ is also gaining in content knowledge.

The first three years of CAST demonstrated that a regional infrastructure was needed to provide training and support and to establish a learning community. The MSC seemed to be the ideal partner. Leveraging the existing professional development system in southwestern Pennsylvania to bring computational science into the curriculum became our goal. We are now in the first year of implementing this strategy.

When the PSC contacted the MSC about including computational science modules in their annual training materials, they were very interested. At an introductory workshop [15], the MVHS team demonstrated three modeling approaches: agent-based, aggregatebased, and interactive spreadsheets. An interactive spreadsheet [16] illustrating coin flipping served as an introduction to the role that random numbers play in simulating real world events. Shodor's Forest Fire applet [17] was used to illustrate the role of probability in an agent-based model. Then a Vensim model of a forest fire spreading [18] was demonstrated to show the similarities and differences between a probabilistic and a deterministic approach to a problem. Since the role of computational thinking in math and science education was already being discussed in the MSC through the Math Science Partnership Circles, the CAST workshop training model proposed was received with open arms. The MSC was as eager to learn how to teach computational thinking and reasoning skills to area science and math teachers as the CAST team was to help them do so.

The next step was to define the organizational structure of the partnership. The PSC was in charge of fund-raising to make the CAST Professional Development Program (CAST-PDP) possible, the MSC was to provide the instructional specialists who would learn how to train and support teachers in using computational science, and MVHS would provide the training materials and the initial training to prepare the MSC for their role. Once the PSC had obtained funding from two Pittsburgh area foundations (DSF Charitable Foundation and the Frick Fund of the Buhl Foundation) to support the conversion of CAST workshop materials into well-defined modules, the partners began meeting to learn how to package the materials to fit the MSC's standard training methodology. After conducting walkthroughs of seven of the twelve modules, the MVHS team modified their materials to include details that the MSC trainers requested. During the summer of 2011, we piloted these modules with groups of teachers and trainers to receive more feedback and to further refine the modules.
The twelve CAST modules are designed to be used in various configurations to support those who wish to focus on using prebuilt models as well as those who wish to become experts in one or more tools. The first group of modules already piloted (1, 2, 3, $5,7,10$, and 12) are designed for model users [15]. The remaining modules, which focus on model building, will be piloted in 2012 .

The titles of the modules are:

1. Introduction to Computational Reasoning

2. Deriving a Mathematical Model: An Experimental and Virtual Approach via Spreadsheets

3. Turning Multivariable Models into Interactive Animated Simulations

4. Building Interactive Excel Simulations

5. Introduction to Agent Modeling

6. Building an Agent Model with NetLogo

7. Introduction to Systems Modeling

8. Building a Systems Model with Vensim

9. Time-Based Models in Excel

10. Comparing Model Environments

11. Choosing a Model Environment to Build Your Model

12. What's Out There: Readily Available Models

\subsection{Provide Materials Relevant to Local Learning Goals}

When MVHS started in 1994, learning goals and expectations varied from one science classroom to another. We had to talk to the teachers in our project to find out what topics were common across all schools and build models addressing the associated concepts. Once MSDE established core learning goals for the sciences, it became easier to identify the computer models and simulations that would be relevant to all science teachers. Thanks to the recent release of the Common Core State Standards [2], computational science educators can now expect that models and simulations developed for one state will be applicable to other states. The fact that there are numerous resources available [19] means that those wishing to work with $\mathrm{K} 12$ school districts have numerous models and simulations to draw from. However, going to the trouble of finding a selection of models that will have your particular audience experiencing "ah-ha" moments will ensure that your message is heard and embraced.

For the CAST-PDP project, the MVHS trainers began by selecting pre-built models that had been shown to be classroom relevant through their use in other teacher training workshops. These models were then linked to specific goals from the Pennsylvania Math and Science Standards [20], and they were demonstrated to the MSC science and math coordinators to assess their impact. If the instructional leaders had "ah-ha" moments, the models were deemed worthy of inclusion in the modules. On the other hand, if a model seemed too confusing or uninteresting, it was either significantly modified or abandoned in favor of a more compelling topic. The models currently in use are available at http://www.psc.edu/eot/k12/2011yr.php.

Another challenge in the development of materials to help teachers infuse computational science in their classrooms is the pedagogy that needs to accompany the models. It isn't enough to know where to find the tools. One also needs to know how to use the tool effectively. In the CAST-PDP project, we demonstrate an inquiry-based pedagogy with every model we include in our modules. Our goal is to prepare teachers to be critical users of computational science tools so that they know how to elicit both computational and conceptual learning from their students. 


\subsection{Provide Opportunities for STEM Teachers to Work Together}

Workshop leaders addressing a mixed audience of science and math teachers have a challenging task ahead of them. The silobased system of college majors has resulted in a lack of communication among biology, chemistry, physics, earth/space science, and math teachers except in very small schools where one person teaches multiple subjects. But, teachers are like students in that they construct their knowledge through interaction with peers, applying their ideas in the classroom, discussing results to refine their understanding, and extending their learning to new situations [21]. Research has shown that richer instruction and improved student learning occur when science, math, technology and engineering teachers and professionals collaborate [22].

The MVHS CoreModels project found that explicit teaching of math terminology helped the science teachers to use those terms in their classroom, thereby helping their students make a connection between math and science. Linear growth, exponential growth or a J-curve, bounded growth or an S-curve - all of these terms have their place in math and science. It is important to make the teachers aware of differences and similarities in terminology so they can help their students see the connections. And, science teachers were often surprised to learn that similar graph shapes were found across their specialty areas. Moreover, the realization that similar graph shapes resulted from similar model structures was a revelation to them. These crossovers between mathematics and the sciences gave the teachers a common ground for discussion about issues of pedagogy and student learning

The current southwest Pennsylvania Math Science Partnership (MSP), facilitated by MSC, creates professional learning communities (PLCs) both within districts, and regionally. Via MSC, the CAST modules will be introduced within the regional learning communities, in order for those leaders to take their understandings back to their building based PLCs. That will enable more than 100 teachers, who are already involved in strengthening their teaching of mathematics and science to consider computer modeling as a new strategy. These regional professional learning communities meet 8 days each school year. The schedule for the 2011-2012 school year begins with the introductory CAST module, facilitated by MSC coordinators who have been trained by the CAST team and who have contributed to the module development. Two additional PLC days will be devoted to additional CAST modeling modules, this time presented centrally in the context of a regional conference bringing all 120 PLC participants to the Carnegie Science Center. Those sessions will be presented by the MVHS team, as the MSC team continues to build capacity for on-going support. The other five PLC sessions will allow the participants to integrate their learning into the on-going strengthening of their practice, supported locally by the MSC Coordinators. In addition, ten day summer institutes, supported by the MSP funding, will feature CAST modules, as an integrated means of exploring life sciences with school year follow-up allowing continuing support.

Our hope is that the science teachers from the school districts in the Pittsburgh area will evolve into self-sustaining PLCs in which teachers share their methods for using computational science in their classrooms. We have already seen a successful example of this in the WCPS district in Maryland where science teachers at both the middle and high school levels incorporate modeling into their instruction. The 2010-11 school year began with a new series of middle school science textbooks which directed students to use a variety of modeling tools, some free like NetLogo [23], others requiring costly GIS software. To enable the full use of the textbook without additional expense, the district-level science instructional specialist collaborated with the teachers to develop interactive web pages incorporating the pertinent NetLogo applets along with the GIS capabilities of NetLogo. At various points during the year, prior to the units in which the models would be used, teachers participated in professional development workshops designed to familiarize them with the models and the applet format. When asked, the science specialist co-taught lessons with teachers as they were using the model applets with students. Based on the statistics option built into the wiki, it is clear that teachers and students are using the models on a regular basis to support student learning.

At the high school level, WCPS is seeing the active use of simulations across the sciences. Physics teachers use the PhET [24] site on a regular basis and some are still using Interactive Physics [25] in their classrooms. Biology teachers are using a sequence of NetLogo based web pages developed by the science specialist to help students with concepts of genetics [14]. This kind of systemic change happens only through consistent opportunities for collaboration.

\section{CONCLUSION}

Preparing teachers to infuse computational science into their classroom instruction is hard work that requires patience, persistence, flexibility, creativity and time. To reach beyond the subset of teachers who actively seek new learning experiences to influence those who are comfortable with the status quo requires the confluence of several factors, including motivation to learn new skills, access to well-designed training materials, local professional development support, and a collaborative environment. External motivation in the form of national standards is now available through the Common Core State Standards which include this goal for grades 11-12 in science: "Synthesize information from a range of sources (e.g. texts, experiments, simulations) into a coherent understanding of a process, phenomenon, or concept, resolving conflicting information when possible" [26]. At this time, all but six states have adopted these standards [27]. The National Research Council's new K-12 science framework includes systems and system models as crosscutting concepts throughout science and engineering [28]. The years of experience that MVHS and others in the computational science education community have accumulated has resulted in an abundance of models, simulations, and training methods available for use. The challenge is forging the alliances with local professional development leaders so those materials can be disseminated more broadly. Finally, as more schools embrace the philosophy behind professional learning communities [29], teachers will feel empowered to try new approaches, knowing that they will have the freedom and support to experiment until they have achieved mastery. We believe that the time is right for advancing the integration of computational science into classroom instruction.

\section{ACKNOWLEDGEMENTS}

The authors wish to thank Dr. Robert M. Panoff, Executive Director and President of the Shodor Education Foundation, for the resources and inspiration he has provided the computational science education community. We also thank Sandy Graff, Supervisor of Secondary Science for the Washington County Public Schools, for her years of support of MVHS initiatives in 
WCPS science classrooms. In addition, we wish to thank all of the Maryland and Pittsburgh-area educators whose participation in and feedback for workshops held over the years have made our current program possible. The following funding agencies provided the support that made this work possible: The National Science Foundation, the DSF Charitable Foundation, the Frick Fund of the Buhl Foundation, the Grable Foundation, and the Heinz Endowments.

\section{REFERENCES}

[1] Honey, M and Hilton, M, eds., Learning Science Through Computer Games and Simulations, The National Academies Press, Washington DC (2011) http://www.nap.edu/catalog.php?record_id=13078

[2] Common Core State Standards http://www.corestandards.org/Accessed August 12, 2011

[3] MVHS website http://mvhs.shodor.org/origins/orindex.html Accessed August 12, 2011

[4] Killion, J. and Harrison, C. Taking the Lead, National Staff Development Council, Oxford, Ohio (2006)

[5] MVHS Core Models http://mvhs.shodor.org/coremodels/cmindex.html Accessed August 12, 2011

[6] Friedman, W. and Culp, K., Evaluation of the CoreModels project: Final report, Education Development Center/Center for Children \& Technology, New York, New York, (2001)

[7] CAST website http://www.psc.edu/eot/k12/cast.php Accessed August 12, 2011

[8] MSDE website http://mdk12.org/assessments/high school/index.html Accessed August 12, 2011

[9] MVHS school list http://mvhs.shodor.org/origins/MVHSSchools.html Accessed August 12, 2011

[10] MVHS Collaborative Projects list http://mvhs.shodor.org/mvhsproj/projects/collab.html Accessed August 12, 2011

[11] CAST 2006 link http://www.psc.edu/eot/k12/first year.php Accessed August 12, 2011

[12] MSC website $\mathrm{http}: / / \mathrm{www}$.aiu3.net/Level2.aspx?id=480 Accessed August 12, 2011

[13] St. Mary's County website http://www.somdtoday.com/2011/04/15/skinner-selected-assmcps-teacher-of-the-year/ Accessed August 12, 2011

[14] Butterfly Genetics website http://web.me.com/scitrout/NetLogo $\% 20$ Models/butterflyintr o.html Accessed August 12, 2011
[15] CAST Modules http://www.psc.edu/eot/k12/2011yr.php Accessed August 12, 2011

[16] Flipping Pennies http://academic.pgcc.edu/ $\sim$ ssinex/excelets/flipping pennies.X 1s Accessed August 12, 2011

[17] Shodor's link to Fire!!! http://www.shodor.org/interactivate/activities/Fire/ Accessed August 12, 2011

[18] MVHS link to Forest Fire page http://mvhs.shodor.org/mvhsproj/forestfire/forestfire.html Accessed August 12, 2011

[19] Computational Science Education Reference Desk http://www.shodor.org/refdesk/ Accessed August 12, 2011

[20] Pennsylvania State Standards for Math and Science http://www.pdesas.org/Standard/StandardsDownloads Accessed August 12, 2011

[21] Krajcik, J.S., Blumenfeld, P.C., Marx, R.W., and Soloway, E., A collaborative model for helping middle grade science teachers learn project-based instruction. Elementary School Journal, 94, 483-497 (1994).

[22] STEM Teachers in Professional Learning Communities http://www.wested.org/online pubs/1098-executivesummary.pdf Accessed August 12, 2011

[23] NetLogo http://ccl.northwestern.edu/netlogo/ Accessed August 12, 2011

[24] PhET http://phet.colorado.edu/ Accessed August 12, 2011

[25] Interactive Physics http://www.designsimulation.com/ip/index.php Accessed August 12, 2011

[26] Common Core State Standards for Literacy in History/Social Studies, Science, and Technical Subjects, page 62, http://www.corestandards.org/assets/CCSSI_ELA\%20Standa rds.pdf Accessed August 12, 2011

[27] Common Core State Standards Adoption Status http://www.corestandards.org/in-the-states Accessed August 12, 2011

[28] Committee on Conceptual Framework for the New K-12 Science Education Standards; National Research Council, A Framework for K-12 Science Education: Practices, Crosscutting Concepts, and Core Ideas, The National Academies Press, Washington DC (2011) http://www.nap.edu/openbook.php?record id $=13165 \&$ page $=$ $\underline{\mathrm{R} 1}$

[29] DuFour, R., Schools as Learning Communities. Educational Leadership, 61, 6-11, (2004). $\mathrm{http} / / /$ pdonline.ascd.org/pd online/secondary reading/el2004 05 dufour.html 